\title{
Clofazimine and Eosinophilic Enteritis
}

\author{
G. H. MASON, R. B. ELLIS-PEGLER AND J. F. ARTHUR \\ Department of Pathology, and Infectious Diseases Unit, \\ Auckland Public Hospital, Auckland, New Zealand
}

\begin{abstract}
A case of eosinophilic enteritis associated with clofazimine (Lamprene) therapy is reported. A 29-year-old Samoan woman with leprosy developed abdominal pain after 3 years of clofazimine therapy at $600 \mathrm{mg}$ daily. At laparotomy there was nodular thickening of the upper ileum with black-brown pigmentation of the ileal wall, mesentery and mesenteric lymph nodes. Ileal biopsy showed eosinophilic enteritis, and red crystals of clofazimine were present in unstained sections of the small bowel mucosa and sub mucosa, as well as in mesenteric lymph nodes. It seems that these histological changes and her peripheral eosinophilia represent a reaction to the drug. The potential hazards of long-term high dose clofazimine therapy are stated again.
\end{abstract}

\section{Introduction}

Jopling (1976) recently reviewed the side-effects of clofazimine in an Editorial in this journal. He stressed those affecting the gastro-intestinal tract. Abdominal pain, anorexia, vomiting, diarrhoea and weight loss have been most commonly documented. Diarrhoea has been seen in patients after only 3 weeks therapy (Imkamp, 1968). Abdominal pain seems to have developed only after more prolonged therapy and only in those receiving daily dosage. Some evidence suggests these effects may be dose related (Plock and Leiker, 1976).

Patients with abdominal symptoms have died soon after this drug has been stopped. In these patients, and in others taking the drug who have come to laparotomy for similar symptoms (Jagadeesan et al., 1975), many tissues have been discoloured. Localized areas of thickening of the small bowel have been described (Karat, 1975). Clofazimine crystals have been demonstrated in gut-wall tissues and mesenteric nodes as well as in other extra-intestinal sites (Desikan et al., 1975). An assessment of the total amounts of drug which can accumulate during treatment has recently been published (Desikan and Balakrishnan, 1976). Histological reaction to the crystals has generally been described as non-specific in type.

We describe here a patient with a predominantly eosinophilic and histiocytic reaction to the drug with peripheral eosinophilia. 


\section{Clinical Details}

A 23-year-old Samoan woman presented to the Infectious Disease Unit, Auckland Public Hospital, Auckland, New Zealand in 1970. When 13 and 14 years old, she had probably received dapsone for leprosy in Samoa. She had widespread skin nodules and a skin biopsy confirmed the diagnosis of leprosy (lepromatous end of spectrum).

She was initially treated with rifampicin alone. However because of frequent type II reactions, prednisone, and clofazimine at $600 \mathrm{mg} / \mathrm{day}$, were begun in 1972. In view of her erratic drug taking no further rifampicin was prescribed after 1974, and she remained on this same dose of clofazimine until 1975.

In early 1975 she developed periumbilical post-prandial pain. There was no diarrhoea. She had a peripheral eosinophilia (maximum of $3600 / \mathrm{mm}^{3}$ ). Small bowel X-rays showed changes in the proximal jejunum which worsened distally. Dilatation of the lumen and coarsening of the mucosal pattern proximally became an irregular luminal calibre and a frankly cobblestone mucosa in the ileum. Tests for malabsorption were normal. No microfilarial or gut parasites were seen on repeated search and chest X-ray was normal. Rectal and peroral jejunal biopsies were done (see below).

With continuing increasing abdominal pain suggesting subacute bowel obstruction, she had a laparotomy in October, 1975, some 6 months after the onset of her symptoms. At laparotomy omentum, small bowel and mesentery were deeply pigmented brown. The jejunum and lower ileum appeared grossly normal but in the upper ileum there were about 20 nodular areas of thickening in the bowel wall. These areas were on both mesenteric and antimesenteric borders and involved only small segments of the total bowel circumference. There was slight inflammation of the overlying serosa. The mesentery contained lymph nodes measuring up to $2.5 \mathrm{~cm}$ across; they were almost black. An ileal "nodule" and a mesenteric node were biopsied and incidental appendicectomy performed. No acid-fast bacilli were subsequently cultured from these biopsies.

A diagnosis of eosinophilic enteritis was made histologically and postoperatively prednisone $60 \mathrm{mg} /$ day was started. Symptoms and peripheral eosinophilia rapidly resolved and clofazimine and prednisone dosages subsequently decreased. Clofazimine was entirely withdrawn in April, 1976 and thiambutosine replaced it. As prednisone has been reduced she has had 2 further episodes of more severe abdominal pain in April and October, 1976. The second of these was again associated with peripheral eosinophilia. Small bowel X-rays in April, 1976 showed the same but less marked abnormalities as previously. Both episodes responded dramatically to increased prednisone dosage. Between these severe bouts of pain, she has had intermittently less defined abdominal pain controlled with simple analgesics.

\section{Pathological Findings (Figs 1-3)}

The initial peroral jejunal biopsy (4.9.75) (Fig. 1) showed an abnormal villous pattern with shortening and broadening of the villi and an increased inflammatory cell infiltrate in the lamina propria. In the submucosa there were prominen: histiocytes; a few of these were multinucleated. Special stains for M. leprae were negative and there was no amyloid in the biopsy.

The full-thickness small bowel biopsy (Figs 2 and 3) taken at laparotomy 


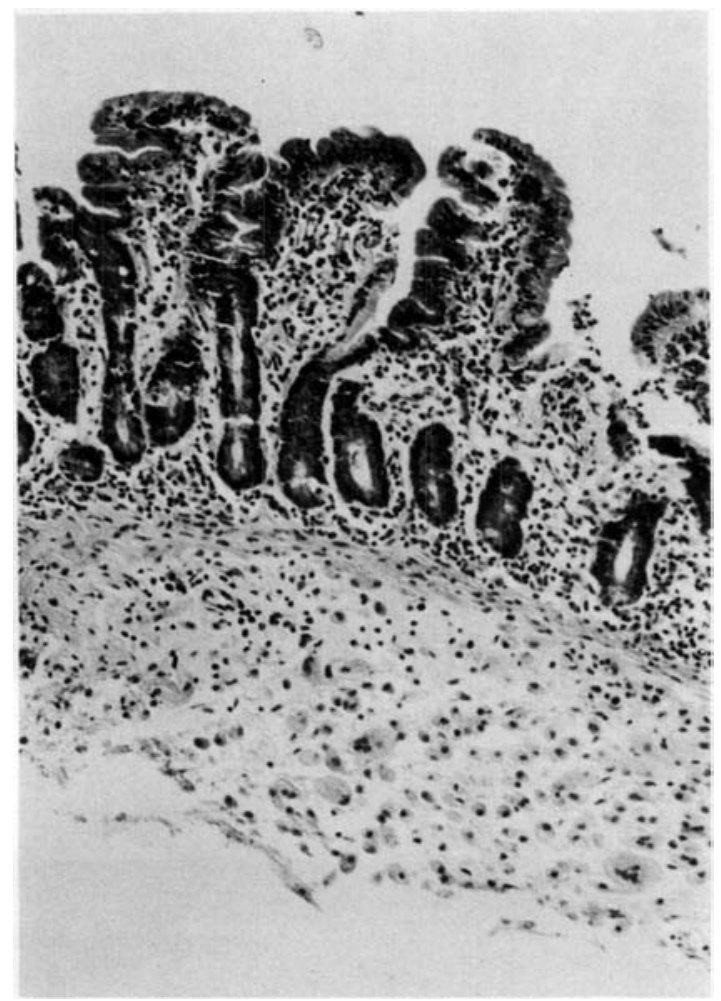

Fig. 1. Per-oral jejunal biopsy showing focal broadening of villi with prominent submucosal histiocytes.

(21.10.75) showed the villous architecture to be normal, although there was some broadening of a few villi. Within the deeper part of the lamina propria and the superficial submucosa there was a dense cellular infiltrate of eosinophils and histiocytes. A sparse infiltrate of eosinophils was also present in the muscle layers. The mesenteric lymph node showed retention of the basic follicular architecture, but within the node there were focal abscesses confined to the paracortical zone and medulla. These abscesses had centres packed almost exclusively with eosinophils; many of them were degenerate. They were surrounded by a zone of histiocytes with occasional eosinophils but no giant cells were present. The sinuses and medullary cords also contained numerous eosinophils.

The histological appearances of the small bowel biopsy fitted well into the described pattern of eosinophilic enteritis. The eosinophilic lesions in the lymph nodes were unusual and their presence suggested the possibility of a more widespread process.

Sections of both the small bowel biopsy and mesenteric lymph node were sent to Dr D. J. Harman of the Leprosy Study Centre, London, and he drew our attention to the presence of clofazimine crystals within both biopsies in the unstained sections. 


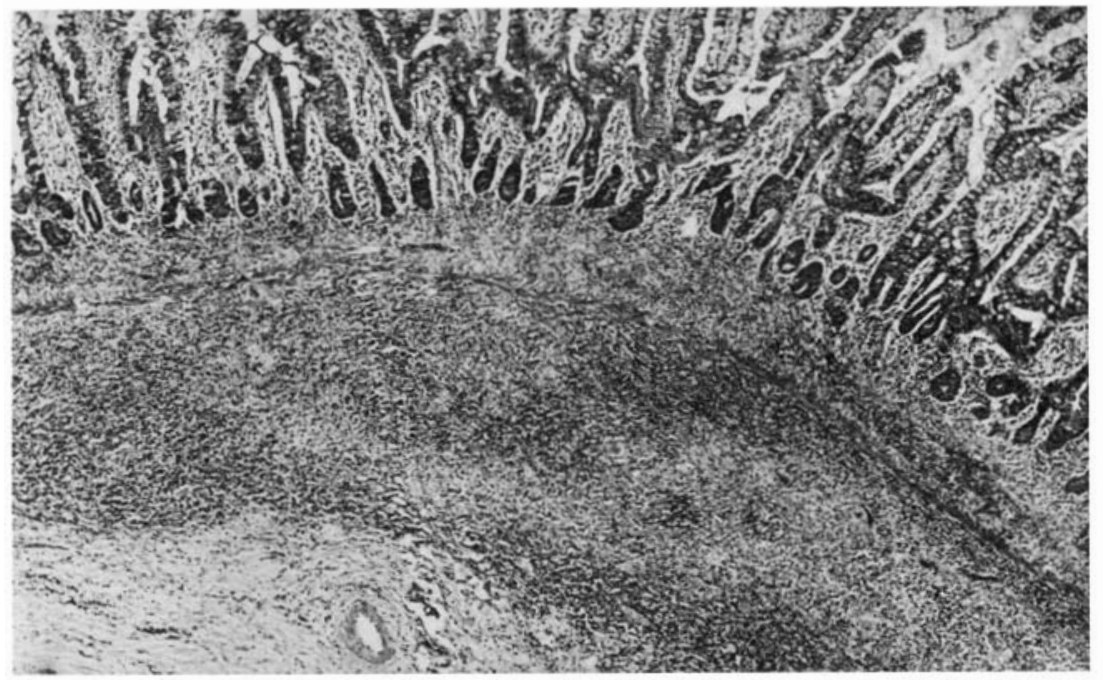

Fig. 2. Full-thickness ileal biopsy showing marked submucosal infiltrate of eosinophils and histiocytes. H. and E. $\times 28$.

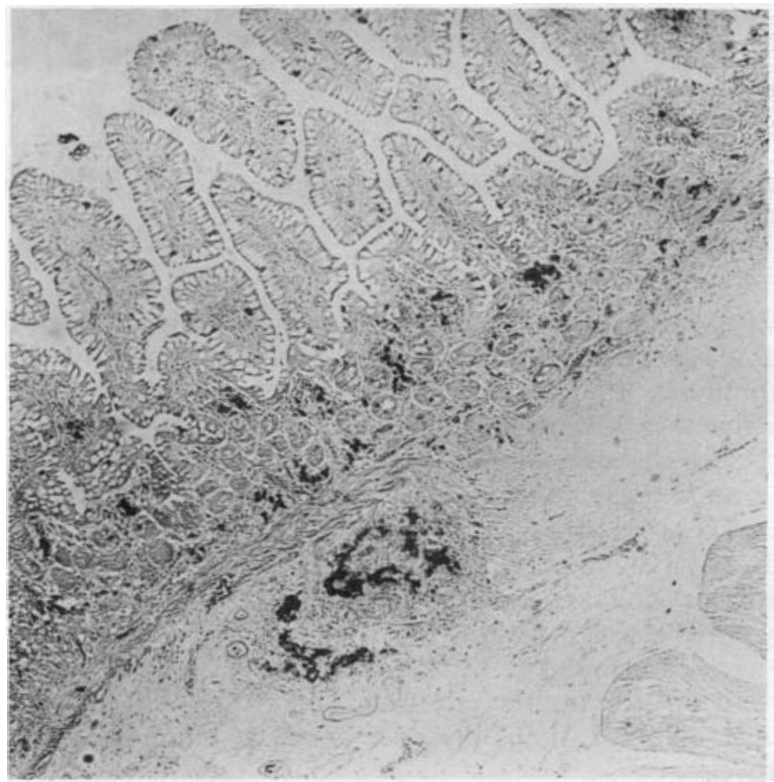

Fig. 3. Full-thickness ileal biopsy showing mucosal surface with black crystal deposition in the deeper lamina propria and superficial submucosa. Picric acid. $\times 32$. 
Clofazimine is alcohol soluble and is thus removed by alcohol used in both processing tissue and in staining the slides. Surprisingly however, enough had remained within the laparotomy biopsies (presumably a reflection of the size of the tissue blocks), to be seen in unstained sections. This red crystalline material present in the deeper lamina propria and superficial submucosa was related anatomically to the heavy eosinophilic and histiocytic infiltrate present in the small bowel biopsy and accounted for the pigmentation seen grossly. Similar crystals were concentrated in the mesenteric lymph nodes at the sites of the focal abscesses described.

The original per-oral jejunal biopsy was re-examined but no crystals were found. This was probably because the crystals dissolved during the processing of this small biopsy. We have assumed that the submucosal histiocytic response was related to crystal deposition. The appendix and the previous rectal biopsies were examined for crystals but none were found. The appendix and the rectal biopsies did however show melanosis coli, and ceroid pigment deposition has been noted before in association with clofazimine treatment (Ridley, quoted by Jopling, 1976). Ceroid pigment was not prominent in the small bowel biopsies. We carried out spectrophotometric examination of an alcoholic extract of clofazimine and of a similar extract of the jejunal biopsy. Similar absorption peaks at about 220, 285 and $450 \mathrm{~nm}$ for both samples confirmed the presence of clofazimine in the biopsy.

\section{Discussion}

Eosinophilic infiltration of the small intestine is uncommon and can be separated into 3 main entities (Morson and Dawson, 1972). They are eosinophilic enteritis, eosinophilic granulomatous polyp (inflammatory fibroid polyp) and allergic gastro-enteropathy. The latter has been described recently in children and is thought to represent an allergy to milk.

Patients with eosinophilic enteritis have shown either single or multiple thickenings of the pyloric end of the stomach and short or longer segments of the small intestine, producing variable degrees of acute or subacute obstruction. There is usually peripheral eosinophilia, and occasional patients have had concomitant malabsorption or protein losing enteropathy. Suggested causes range from food sensitivities e.g. onions and chocolate, to parasite ingestion and infestation.

Only one other patient taking clofazimine has had eosinophilic infiltration of the gut noted on histological examination (Jagadeesan et al., 1975) and peripheral eosinophilia has not previously been reported. Eosinophils were not found in animal tissues in early studies of the drug (Conalty, quoted by McDougall, 1976). Nevertheless the apparent anatomical relationship of the drug to the tissue eosinophils and the exclusion as far as possible of other causes of intestinal eosinophils and peripheral eosinophilia make the causal association in this case quite strong.

Fourteen months after initial reduction in dosage to $100 \mathrm{mg} 3$ times per week, and 6 months after completely stopping clofazimine this patient still has abdominal symptoms suggesting continuing reaction to it. Clofazimine crystals have been found present in lymph nodes almost 4 years after the drug was stopped (Jopling, 1976). The use of this agent at high dosage for lengthy periods is thus clearly contra-indicated if potentially toxic tissue accumulation is to be avoided. Life-threatening toxic effects have not been described in patients who 
have taken only the low clofazimine dosage which is recommended for its anti-leprotic effect. Those who would use it for its "anti-reactional" properties must be aware of this potential long term toxic effect if used excessively-as, in retrospect, it was in this case.

\section{Acknowledgements}

We would like to thank Drs W. R. Lang and C. Howden from the Infectious Diseases Unit, Mr J. Carter of the Department of Surgery, and the Departments of Histopathology, Chemical Pathology, Radiology and Photography, Auckland Hospital for their help. We would also like to thank Dr D. J. Harman of the Leprosy Study Centre, London for his major contribution to the ultimate diagnosis.

\section{References}

Desikan, K. V. and Balakrishnan, S. (1976). Tissue levels of clofazimine in a case of leprosy. Lepr. Rev. 47, 107.

Desikan, K. V., Ramanujam, K., Ramu, G. and Balakrishnan, S. (1975). Autopsy findings in a case of lepromatous leprosy treated with clofazimine. Lepr. Rev. 46, 181.

Imkamp. F. M. J. H. (1968). A treatment of corticosteroid-dependent lepromatous patient in persistent erythema nodosum leprosum; a clinical evaluation of G.3032 (B.663). Lepr. Rev. 39, 119.

Jagadeesan, K., Visweswaran, M. K. and Harihara Iyer, K. (1975). Acute abdomen in a patient treated with Lamprene. Int. Surg. 60, 208.

Jopling, W. H. (1976). Editorial. Complications of treatment with clofazimine (Lamprene: B663). Lepr. Rev. 47, 1.

Karat, A. B. A. (1975). Long term follow-up of clofazimine (Lamprene) in the management of reactive phases of leprosy. Lepr. Rev. 46 (Suppl.) 105.

McDougall, C. (1976). Personal communication.

Morson, B. and Dawson, I. M. P. (1972). Gastrointestinal Pathology. Oxford: Blackwell Scientific Publications.

Plock, H. and Leiker, D. L. (1976). A long term trial with clofazimine in reactive lepromatous leprosy. Lepr. Rev. 47, 25. 